Service social

\title{
Leçons à tirer de nos traditions en service social des groupes
}

\section{Margot Breton}

Volume 39, numéro 1, 1990

Le groupe ici et ailleurs

URI : https://id.erudit.org/iderudit/706453ar

DOI : https://doi.org/10.7202/706453ar

Aller au sommaire du numéro

Éditeur(s)

École de service social de l'Université Laval

ISSN

1708-1734 (numérique)

Découvrir la revue

Citer cet article

Breton, M. (1990). Leçons à tirer de nos traditions en service social des groupes. Service social, 39(1), 13-26. https://doi.org/10.7202/706453ar

\section{Résumé de l'article}

L'article s'inspire des trois sources principales du service social des groupes : le mouvement des "settlements ", celui de la " récréation » et celui de " l'éducation progressiste ". S'appuyant sur le double intérêt de cette profession pour l'individuel et le collectif, l'auteure discute et actualise les principes qui ont orienté les trois mouvements précités, et donne un aperçu des défis qu'a à relever le service social des groupes dans notre monde complexe et en état de changement rapide. 


\section{ARTICLES}

Margot Breton, professeure à la Faculté de service social, Université de Toronto.

\section{Leçons à tirer de nos traditions en service social des groupes*}

Margot Breton

L'une des traditions les mieux conservées en service social des groupes est celle, justement, de tirer parti de nos traditions, en remettant en cause et analysant notre passé (Alissi, 1983; Garvin, 1984; Schwartz, 1986; Lee et Swenson, 1986). L'importance accordée à la tradition et à son impact sur la pratique actuelle du service social des groupes remonte aussi loin que 1955, alors que Trecker publiait son ouvrage intitulé Group Work: Foundations and Frontiers. La deuxième partie de son livre rapporte les réflexions de travailleurs sociaux de groupes sur l'avenir de cette méthode et sur la nécessité de faire face aux défis qui lui sont lancés dans un monde en constants changements et transformations. Plusieurs passages de ce livre sont encore d'actualité mais j'en retiens particulièrement deux. Le premier est un avertissement et se lit ainsi : "Notre tâche actuelle doit être celle de réexaminer, de clarifier et d'augmenter l'efficacité de notre pratique professionnelle, en mettant de côté, si nécessaire, des éléments familiers de notre passé, pour ajouter et incorporer les apprentissages nouveaux et les progrès qui ont été réalisés ». Le second passage représente une mise en garde : "La claire identifica- 
tion de nos difficultés professionnelles ne progressera que si nous nous attachons aux éléments les plus sains de ce que nous avons appris... et si nous voyons à ce que cet acquis soit encore enrichi. Mais cet enrichissement ne peut provenir uniquement des autres spécialités professionnelles...» (Trecker, 1955 : 377).

Cette déclaration en deux points suppose que l'on considère la tradition non pas comme la préservation du passé, mais comme la manifestation, dans le présent et sous différentes formes, d'idées et de comportements auxquels on attache du prix; quelque chose plus près du concept grec d'entéléchie - l'esprit qui informe - que des concepts d'imitation ou de répétition. Nous devons nous rappeler que nous perdrons les caractéristiques propres au service social des groupes si nos innovations ne s'inspirent que d'autres spécialités et négligent notre propre base d'expériences. Le présent article s'inspire des trois sources principales de la pratique du service social des groupes : le mouvement des "settlements» (The Settlement Movement), celui de la " récréation " (The Recreation Movement) et celui de "l'éducation progressiste" (The Progressive Education Movement), appelé aussi mouvement de l'éducation nouvelle ou de l'école nouvelle.

\section{Le mouvement des settlements}

Les pionniers de ce mouvement avaient délibérément choisi de considérer les gens non seulement comme des personnes, mais aussi comme des membres de cultures et de groupes sociaux influencés par les conditions sociales, économiques et politiques dans lesquelles ils étaient insérés. À cette position "intellectuelle», ils ajoutèrent celle - morale - de susciter la transformation des conditions de vie qu'ils estimaient injustes. Ils choisirent enfin d'adopter la stratégie d'aider les gens à s'efforcer d'améliorer eux-mêmes leurs conditions et de faciliter les actions et les conduites propres à réaliser ce but. On reconnaît là les trois étapes du "voir, juger, agir » qui ont marqué l'action des mouvements de jeunesse des années trente, aussi bien que celle des actuels " mouvements de libération ". La base et la force des prises de position intellectuelles, morales et stratégiques des travailleurs des settlements étaient constituées par leur désir sincère d'abaisser les barrières existant entre les classes sociales et les cultures. À cette fin, "ils établissaient des liens de véritable amitié avec les pauvres et cherchaient à prendre directement conscience de leur mode de vie » (Schwartz, 1986 : 13). Deux commentaires peuvent être formulés au sujet de cette prise de conscience stipulant que les gens appartiennent à différents groupes et cultures, et au sujet du 
désir d'en connaître davantage sur les personnes, les groupes sociaux et les cultures.

II est certes louable de vouloir mieux connaître les personnes et les différents groupes et cultures, mais cela ne suffit pas. Je suis persuadée que la plupart des travailleurs sociaux affirmeraient, par exemple, qu'il ne suffit pas que les hommes soient mieux informés au sujet des femmes, mais qu'il leur faut être informés par les femmes elles-mêmes. Le mouvement de prise de conscience et de libération des femmes nous a éveillés à cette réalité. Cependant, je ne suis pas certaine que nous soyions prêts à adopter ce type de réflexion. Sommes-nous d'accord pour affirmer que les membres d'une culture majoritaire ont besoin de s'informer non seulement de ce qui caractérise une culture minoritaire, mais d'apprendre de cette culture même ? Reconnaissons-nous que les personnes en pouvoir doivent accepter d'apprendre ce qui concerne les sans-pouvoir, mais aussi d'être éclairées par eux? Acceptons-nous que les experts aient besoin de recevoir de l'information de la part des gens ordinaires; et les professionnels d'en recevoir des non-professionnels? Que les travailleurs sociaux ont le devoir d'apprendre ce qui concerne leurs clients, individus ou groupes, mais aussi d'accepter d'être informés par eux ? Nous avons l'obligation de nous poser ces questions. C'est à la seule condition que nous soyions prêts à nous laisser éclairer par le pauvre, le sans-pouvoir, celui qui balbutie, comme par les nonprofessionnels et les membres des groupes minoritaires, que nous exécuterons notre travail dans un esprit réel de partage du contrôle, du pouvoir et de l'utilisation de l'agenda de travail, comme l'a écrit Schwartz (1986 : 24).

La résistance que nous opposons à l'abandon d'une partie de notre "pouvoir professionnel " peut très bien expliquer la quasiinterruption de notre travail auprès des groupes dits " de changement social ». Dans leur analyse de la pratique du service social des groupes à Montréal, Pâquet-Deehy et al. (1985) ont démontré que $7 \%$ seulement de cette pratique s'adresse aux groupes de changement social; elles croient que cette situation traduit la " faible priorité accordée à la réforme sociale dans un contexte de réductions budgétaires" et la "décision des administrateurs de donner la préférence au travail avec les groupes de changement personnel plutôt qu'avec les groupes communautaires d'adultes ». Cependant, comme l'étude en question a démontré que, dans les groupes de changement social, la position centrale et le contrôle du travailleur social sont moins marqués que dans les groupes de thérapie ou de changement individuel, je crois que les décisions administratives reflètent aussi les préférences professionnelles des travailleurs sociaux pour l'exercice de plus, plutôt que moins, de pouvoir. J'ai 
déjà exposé cette question (Breton, 1989) et elle continue à requérir une sérieuse attention.

Notre intérêt pour l'exercice du " pouvoir professionnel » peut nous aider à déceler les deux pôles du service social : "le changement dans les personnes » et «le changement dans les systèmes " (Alissi, 1983). Alors que notre propre image d'humaniste nous porte à rechercher le "changement dans les systèmes", notre moi professionnel nous incite à désirer le changement des personnes. Mais la compréhension est une chose et l'atténuation de la polarisation en est une autre. Sous l'influence de la stratégie chère au travailleur social du settlement, qui le portait à engager les personnes victimes de conditions injustes à lutter pour le changement social, ainsi qu'à la lumière des approches et méthodes contemporaines, je propose que l'éveil de la conscience devienne un but recherché dans toutes les formes de pratique du service social. Je ne veux pas dire par là que tous les groupes devraient être structurés comme les groupes de conscientisation, mais que les principes de la conscientisation devraient animer toute notre pratique. De cette façon, le changement ne serait pas pensé uniquement en termes de changement dans l'individu, qu'il s'agisse des attitudes, des processus de pensée ou des comportements; on accorderait autant d'importance aux changements sociaux, économiques et politiques qui touchent les personnes en tant que membres de groupes sociaux, de races, de classes, de cultures et de sexes. Nous pouvons aussi, dans le même esprit, nous inspirer de la pédagogie contemporaine de la libération (Freire, 1973) et axer notre pratique sur l'augmentation du pouvoir, plutôt que sur l'assistance ou sur l'aide aux personnes. Nous considérerons alors les membres de nos groupes comme des sujets actifs plutôt que comme des récipiendaires d'aide, et les encouragerons à se voir tels. Ce mode de pensée fait partie de la tradition du service social des groupes, mais en un temps où de plus en plus de travailleurs sociaux se laissent encore attirer par "l'auréole » de la pratique clinique (Trecker, 1955 : 399), on ne doit pas croire que le principe de "l'accroissement de la capacité »soit acquis.

Quant à la conviction nous venant du mouvement des settlements que les personnes sont reliées à des groupes sociaux et à des cultures propres, je présume que la plupart des travailleurs sociaux d'aujourd'hui pensent en termes de "systèmes" et sont bien conscients de l'influence exercée par des systèmes de plus grande envergure sur les individus, les familles et les petits groupes. Cependant, à l'encontre des travailleurs de settlements d'autrefois, nous ne mettons pas toujours ces connaissances à profit pour nous intéresser aux plus vastes systèmes, nous engager nous-mêmes au niveau politique et engager nos groupes dans des mouvements de 
changement social et politique. Nous utilisons plutôt la théorie des systèmes pour mieux comprendre, évaluer et effectuer des changements chez les individus, les familles et les petits groupes.

Cette façon différente de faire s'explique par le fait que " nous avons appris à nous spécialiser en fonction du nombre " (Schwartz, 1971 : 19), et que, malgré nos prises de position sophistiquées au sujet des systèmes, la plupart d'entre nous confions les questions d'envergure "macroscopique » aux spécialistes des politiques et de la planification, et renvoyons les situations d'envergure communautaire aux spécialistes de ce domaine. (Lewis, 1983, a cependant signalé de notables exceptions à cette façon d'agir). Quoi qu'il en soit, il existe peut-être une explication plus profonde à cette situation. II faut se souvenir que les premiers travailleurs des settlements adoptaient un point de vue moral lorsqu'ils estimaient que certaines situations étaient injustes et ils appuyaient leur indignation d'actions destinées à corriger ces injustices sociales. De notre côté, nous de l'ère présente avons adopté ce que nous appelons une approche "scientifique »des situations-problèmes et croyons que nous agissons de façon scientifique si nous ne formulons pas de jugements ou si nous nous montrons moralement neutres. À l'exemple des membres de plusieurs autres disciplines, nous hésitons à affirmer que tout paradigme reflète des prises de position morales (Kuhn, 1970). II s'ensuit que malgré nos prises de conscience des maux qui découlent de la pauvreté, du racisme, de la discrimination à l'égard des sexes et de l'élitisme culturel, la lutte contre ces maux tient relativement peu de place dans notre pratique professionnelle. Dans ces conditions, il n'est pas étonnant de voir publier tant de travaux sur la thérapie individuelle ou collective et de voir tant de travailleurs sociaux aspirer à devenir thérapeutes. L'attrait pour le travail clinique provient en partie de la conviction que "les modèles psychothérapeutiques" sont plus "scientifiques » que les modèles psychosocial ou de travail social. Je suis portée à croire, cependant, que nous prenons prétexte d'une préférence pour le travail clinique et pour une approche "scientifique" dans le but de ne pas avoir à nous orienter vers I'action et vers l'engagement politique que supposent certains types de pratique professionnelle.

L'orientation de plusieurs vers la thérapie ne doit pas nous étonner, mais nous inquiéter. Nous devrions, en effet, nous inquiéter de ce que le service social soit en train de devenir " une forme de psychothérapie " (Garvin, $1984: 15)$ et que, par conséquent, le rôle $\mathrm{d}^{\prime}$ agents de changement que les groupes peuvent jouer dans leur environnement soit plus ou moins perdu. Nous devrions nous en inquiéter parce que si notre action ne reflète pas notre préoccupation 
de justice sociale, nous devenons inutiles pour les pauvres, les opprimés, les membres des minorités et les marginalisés.

La présente analyse ne devrait pas être considérée comme un plaidoyer contre la science. Au contraire, en découvrant que nous avons beaucoup perdu de l'orientation traditionnelle des settlements vers l'action, nous devons nous mettre à l'œuvre et chercher un moyen inventif de concilier "I'approche scientifique ou objective" avec l'engagement de portée morale par lequel nous identifierons et évaluerons les maux sociaux et les combattrons par des actes aussi bien que par des paroles. À ces fins, nous devrons accepter de jouer un rôle plus actif de médiation dans le milieu concerné, comme l'a proposé Parsons (1988 : 43). Dans son analyse d'un groupe de filles appartenant à une minorité à faible revenu, elle a fait remarquer que " les travailleurs sociaux doivent apprendre à rejoindre les enfants les plus pauvres et à orienter efficacement vers eux les ressources susceptibles de leur venir en aide...» Je crois que la médiation efficace du travailleur social pour un milieu donné inclut l'engagement politique en tant que partie du paradigme et des stratégies de notre travail pratique; et ceci sous-tend que nous devrons faire face aux confrontations apparaissant nécessairement aux niveaux professionnel, institutionnel et social.

\section{Le mouvement de la récréation}

Si l'action des settlements attire notre attention sur le moi social, le mouvement de la récréation nous rappelle le moi total. II nous remémore le fait que chaque personne humaine possède la capacité de se développer et d'être créatrice. C'est à nos risques que nous négligeons ce rappel. Nous vivons dans une ère où les travailleurs sociaux sont confrontés à des difficultés et des tâches complexes. II semble que le monde actuel soit de plus en plus peuplé de victimes : des enfants victimes d'abus physiques et sexuels; des adolescents incarcérés dans des institutions psychiatriques à la demande de leurs parents, qui leur reprochent de se montrer rebelles; des jeunes qu'on a jetés à la rue et qui se livrent à la prostitution parce qu'ils ont faim; des femmes battues par leurs maris ou compagnons; des familles sans abri qui vivent dans des conditions inhumaines; des hommes et des femmes qui ont besoin de traitements psychiatriques et qu'on abandonne à la rue; de vieilles gens oubliées par tous; des réfugiés dont les espoirs sont trahis, et tant d'autres cas. En présence de toute cette misère, nous pouvons trouver insolite la pensée que notre travail professionnel puisse englober l'utilisation créatrice des activités de loisirs et de récréation. C'est une pensée qui a eu cours dans 
notre histoire ancienne ou, à tout le moins, dans des temps et dans des endroits plus paisibles que ceux d'aujourd'hui. Cependant, nous aggravons la situation de ces "victimes " si nous oublions qu'elles ne sont pas que des victimes. Elles sont aussi des personnes humaines douées, comme toute autre, de possibilités et d'aspirations au développement de leur créativité et ayant le même besoin de "remplir et de réaliser leur vie en s'associant à d'autres êtres humains "(Cohen, 1952 : 198). II est facile de nous intéresser aux difficultés plus qu'à la personne qui les subit, et de trouver la victime plus intéressante que l'être humain. Alors, nous nous contentons de traiter les effets débilitants du mal subi par la victime et tournons le dos à un type préventif de service social des groupes qui promeut la compétence sociale.

Il importe que nous réveillions notre intérêt pour la " prévention primaire et secondaire " qui, selon Middleman (1978:19) a déjà distingué le service social des groupes du service social personnel et ralliait notre " intérêt premier pour la réalisation d'une croissance humaine normale». Si nous acceptons que la croissance ne se termine qu'à la mort, notre intérêt doit s'appliquer à toute la pratique du service social des groupes et non seulement à l'action auprès des enfants. Cependant, j'avoue que je suis préoccupée par notre virtuelle perte d'intérêt pour les enfants et les jeunes " normaux » ou, plus précisément, pour ce qu'il y a de "normal » dans chaque enfant et chaque jeune. Je me demande si, bien maladroitement, nous ne contribuons pas à la négligence dont fait preuve la société à l'égard de cette portion de notre population. Cette négligence constitue une malheureuse caractéristique de notre époque vorace. (À ce sujet, voir Kolodny et Garland, 1984, et Middleman et Goldberg, 1988).

II reste à trouver un équilibre réalisable entre l'attention due à la victime (ou à la personne perturbée, ou souffrante, etc.) et l'attention à accorder à la personne totale. Je suis convaincue que l'un des moyens de parvenir à cet équilibre consiste à faire, de tout groupe de service social, une entité " démocratique et créatrice", comme l'a exprimé Grace Coyle. Dans une communication intitulée "Group Work as a Method in Recreation ", Coyle a écrit : " L'un des principes qui inspirent la méthode du service social des groupes est la conviction qu'une des sources principales de réalisation de soi vient de la satisfaction profonde qu'apportent les interactions vécues dans un groupe démocratique et créateur " (Coyle, 1947, reproduit dans Trecker, 1953 : 96). Je souligne le fait que cette déclaration ne tend pas à supprimer la préoccupation pour les besoins de la victime, mais $q^{\prime}{ }^{\prime}$ elle nous force à considérer aussi les droits de la personne entière. À mon avis, le langage de Coyle s'adresse directement à ceux qui sont engagés dans les luttes contemporaines pour la libération, y 
compris ceux qui ne veulent d'aucune étiquette, car elle déclare que les humains ont le droit de développer leur plein potentiel en tant que personnes et ont aussi le droit de faire l'expérience profondément satisfaisante de la participation à un groupe démocratique et créateur.

Par conséquent, la façon dont un groupe est structuré est très importante. Il peut être structuré de telle sorte que le participant, dans tous les aspects de sa personne, soit invité à y prendre part, ou bien il peut ne faire appel qu'à ce qu'il y a de troublé, de brisé, de blessé dans le participant. Dans le premier cas, chaque membre du groupe peut vivre une expérience d'autoréalisation et de profonde satisfaction. Dans le second cas, il n'en est rien. Nous devons donc trouver des moyens inédits de faire participer toute la personne. Pour cela, nous devons faire table rase des définitions périmées de la motivation (Breton, 1985), nous familiariser avec un modèle d'apprentissage social permettant croissance et changement (Goldstein, 1988), comprendre, respecter et utiliser les caractéristiques culturelles, tout en acceptant, de façon constructive, le mécontentement, le ressentiment et parfois la rage des membres de minorités raciales opprimées (Davis, 1984). Il nous faut aussi trouver des moyens innovateurs de faire de nos groupes, des milieux où les gens peuvent " devenir", c'est-à-dire où ils peuvent "agir " (Middleman, 1983). À ces fins, nous devrons réapprendre à ne pas craindre de recourir à des activités et à y trouver du plaisir; pour cela, nous devrons croire au pouvoir guérisseur et constructif du jeu en lui-même au lieu de ne croire qu'à la thérapie par le jeu. Cette réflexion nous obligera à scruter les présomptions qui ont inspiré, pour une grande part, la pratique du service social des groupes et qui nous ont orientés vers un genre mortellement sérieux de travail avec les groupes. Souvenons-nous que le service social des groupes doit une grande part de sa vitalité et de ses particularités aux théories sur la croissance normale et le développement, ainsi qu'à la préoccupation pour la personne totale, théories qui ont émergé de son association primitive avec le mouvement de la récréation. Pour faire face aux défis que nous lance un contexte social en voie de changement, nous ferions bien de nous appuyer sur les traditions issues de ce mouvement.

\section{Le mouvement de l'éducation progressiste}

Ce mouvement fut influencé par l'avènement des sciences sociales (en particulier, de la psychologie sociale et de la sociologie) et par leurs définitions primitives du petit groupe (Cooley, 1909) et des ressources qu'il présente en éducation (Dewey, 1922). Il a reflété aussi les positions philosophiques de cet auteur sur les modèles 
idéaux de gouvernement et sur le statut de citoyen. Les tenants de ce mouvement (parmi lesquels les travailleurs sociaux de groupe étaient prédominants) considéraient la participation au petit groupe comme une expérience offerte aux personnes pour les préparer à apporter une participation démocratique aux affaires de la communauté. Cette dernière section de l'article est axée sur le petit groupe et sur ses rapports avec la communauté. J'y traite deux questions : a) la définition du pouvoir du petit groupe et la signification que revêt l'aide réciproque; b) la pertinence de la participation démocratique pour la pratique du service social des groupes dans un monde où se côtoient une pluralité de personnes aux cultures originales diverses.

L'intérêt de la sociologie et de la philosophie pour la réciprocité existant entre l'individu et la société et entre le petit groupe et la société, était inscrit dans les premières tentatives de mise en forme du service social des groupes, alors que Grace Coyle (1946) recherchait une base de connaissances englobant aussi bien les relations communautaires que les comportements humains, les processus de groupe, le contenu des programmes et les activités, ainsi que la supervision des praticiens. L'importance à donner aux relations extérieures devint une caractéristique du service social des groupes tant et si bien qu'Alissi (1982 : 15) écrivit qu'à l'encontre des autres méthodes de groupes, " en service social des groupes, le groupe est considéré comme partie naturelle d'un environnement plus vaste et doit, autant que possible, être traité comme tel ». Le fait de considérer le groupe comme étant partie d'un tout plus vaste (point de vue systémique) permet non seulement une meilleure compréhension de ce groupe, mais également de considérer son influence sur la structure sociale plus large (Abels et Abels, 1980).

Au sujet du concept de réciprocité, Schwartz (1961), dans un article intitulé " The Social Worker in the Group », a énoncé ses idées innovatrices sur l'aide mutuelle. Depuis lors, la dynamique et les techniques de l'aide mutuelle dans le petit groupe ont été explorées et ont fait l'objet de plusieurs communications écrites (e.g. Shulman, 1984; Gitterman et Shulman, 1985). L'application des idées de Schwartz à différents contextes de travail social et à des populations diverses est de plus en plus mentionnée dans les publications qui traitent du service social des groupes (e.g. Lee, 1986; Parsons, 1988; Shields, 1986).

Je présume qu'à présent, tout travailleur social qui n'aurait suivi qu'un seul cours en service social des groupes est au moins conscient du fait que le groupe constitue un système d'aide mutuelle. Cela n'a pas toujours été le cas; Shapiro (1977) l'a signalé il y a plus d'une décennie, en soulignant notre négligence à l'égard de ce phénomène. Il semble juste d'affirmer qu'aujourd'hui l'aide mutuelle est perçue 
comme un phénomène " intra-groupe ». On reconnaît le pouvoir que les membres détiennent pour s'influencer les uns les autres et $s^{\prime}$ entraider. La reconnaissance de ce pouvoir est un sine qua non dans le travail efficace avec les groupes. Je crains, cependant, que le fait de réduire l'aide mutuelle à l'état de phénomène intra-groupe nous ait portés à nous concentrer sur le pouvoir de guérison de cette aide mutuelle et à oublier son pouvoir de libération. Celui-ci est relié à la solidarité extra-groupe et intra-groupe, qui produit la force, l'action et le changement aux niveaux social, économique et politique. La solidarité intra-groupe, une expression qui recouvre l'aide mutuelle, conduit à la force, à l'action et au changement au niveau personnel. La préoccupation pour ces trois éléments représente la raison d'être de notre profession, mais elle est tout simplement insuffisante si nous voulons faire œuvre signifiante pour les pauvres, les victimes de préjugés raciaux ou ethniques ou d'abus sexuels, les enfants, les jeunes et les gens âgés qu'on exploite, et tous ceux qui sont marginalisés socialement et aspirent à détenir un peu de pouvoir.

Par conséquent, nous devons mettre au défi notre conceptualisation actuelle de l'aide mutuelle de sorte qu'elle puisse englober tous les aspects de la solidarité : à l'intérieur et à l'extérieur des groupes et entre eux. Nous possédons déjà, pour nous guider en ce sens, des éléments d'un cadre conceptuel plus large, tels que le modèle de vie quotidienne appliqué au service social (The Life Model of Social Work Practice) de Germain et Gitterman (1980), l'approche intégrée (Integrative Perspective) que Lee (1986) et d'autres (Parson et al., 1988) sont à élaborer, ainsi que des analyses historiques et critiques du concept d'aide mutuelle (Lee et Swenson, 1986). En consultant les œuvres de Lang (1986) sur les collectivités, de Schopler et Galinsky (1984) sur les groupes "ouverts", et celles de nombreux professeurs et praticiens sur les groupes de courte durée (Alissi et Casper, 1985), nous pouvons en déduire que la solidarité peut exister dans les systèmes sociaux décrits dans ces textes. Quoi qu'elle soit différente de celle qui se construit dans les groupes bien établis, autonomes et stables, cette solidarité peut être utilisée pour la réalisation de buts sociaux et collectifs aussi bien que pour des fins personnelles. De plus, notre connaissance des modes de création des réseaux en rapport avec le service social des groupes (Abels et Abels, 1980; Shapiro, 1986) nous porte à croire que la solidarité entre les gens représente plus qu'un processus intra-groupe d'aide mutuelle et à y voir le germe du changement social autant que personnel.

En l'absence de ces nouveaux modes de pensée, nos perceptions et nos interprétations de l'aide mutuelle vont devenir de plus en plus narcissiques; et le narcissisme est dangereux dans un monde de cultures pluralistes. Dans cette conjoncture, notre attachement aux 
idéaux et à la participation démocratiques dans et hors le groupe est de grande importance. Mais nous devons aussi être prêts à innover tant au plan conceptuel que pratique. Nous devons réfléchir et nous demander ce que signifie le mot "communauté » dans un monde où se heurtent diverses civilisations, valeurs et cultures, au sein de ce qu'on appelle des villes et villages globaux. II nous faut repenser la "participation aux affaires communautaires " et sa signification pour des tranches de population dont les droits sont bafoués (Breton, 1987). Si nous nous posons ces questions, cela signifie que nous sommes encore intéressés aux causes de la paix et de la justice sociales et non pas seulement à la recherche de la paix de l'esprit et de la santé mentale. Si nous tentons de répondre à ces questions, cela signifie que nous sommes prêts à mettre de côté les interprétations narcissiques de l'aide mutuelle, par lesquelles nous encourageons les membres des groupes à s'occuper de ceux qui sont "dans la même barque " qu'eux, sans réussir à leur faire prendre conscience du fait que certains sont dans "d'autres barques".

\section{Conclusion}

Pour survivre, faire face et se réaliser dans un monde de cultures pluralistes et en état de changement rapide, il nous faut prendre conscience du fait que notre propre intérêt est lié au bien-être du plus vaste système écologique qui nous englobe et non seulement à celui de notre plus proche "niche écologique". Cela exige que nous acceptions de nous accommoder des visées philosophiques, religieuses, économiques, sociales et politiques qui diffèrent de celles de nos propres groupes de référence, sans renoncer aux valeurs fondamentales qui concernent les libertés et droits individuels et collectifs. Il y a là, pour le travailleur social des groupes, un défi que nous pourrons relever après avoir résolu certaines questions.

Tout au long de la présente communication, j'ai présumé que ce qui fait la spécificité du service social des groupes, c'est " son double intérêt pour l'individu et pour la société » (Alissi, 1982 : 10). Étant donné que cette affirmation est partagée par les travailleurs sociaux, il est aberrant que nous nous soyons éloignés des sciences sociales (sciences économiques, sociologie, anthropologie sociale, psychologie sociale), tout comme de disciplines telles que la pédagogie, laissant passer les chances d'influencer la connaissance et de nous laisser influencer par elle. Nous avons choisi, non pas tellement de dialoguer avec la psychiatrie mais de l'écouter et d'écouter surtout la psychiatrie clinique plutôt que la psychiatrie communautaire. J'ai signalé précédemment que l'action en vue de procurer le chan- 
gement social conduit à l'action politique et comporte beaucoup plus de risques, pour les travailleurs sociaux et pour les institutions qui les emploient, que l'action menée en vue de produire le changement personnel. On peut donc prévoir que dans un temps de restrictions comme celui que nous vivons, les efforts consentis pour préserver le statu quo social - et, par conséquent, les approches thérapeutiques - seront encore plus marqués qu'ils ne l'ont été jusqu'ici.

Cependant, si nous devons trouver des réponses innovatrices en service social pour relever les défis que nous rencontrons, je crois qu'il nous faut intégrer le changement social et l'engagement politique à tous nos modèles de pratique. Je pense que l'engagement de certains travailleurs sociaux dans une pratique orientée vers la communauté et l'action sociale ne doit pas suffire à nous donner bonne conscience ou à nous faire accepter que le plus grand nombre de nos praticiens optent pour une forme de travail assimilable à la psychothérapie individuelle ou collective. II est urgent que la recherche en service social des groupes se concentre sur plus que le changement du comportement individuel et sur plus que les processus de petit groupe qui facilitent ce changement.

La tradition du service social des groupes est pour nous une source de sagesse et d'expérience qui nous aidera à harmoniser les actions à poser pour le bénéfice des personnes, du petit groupe et de la communauté. Nous avons donc un rôle-clé à jouer, en tant que profession, pour que le monde nouveau et de plus en plus complexe qui se profile à l'horizon de notre avenir, devienne pour tous et chacun le meilleur milieu de vie possible.

\section{Notes}

* Discours d'ouverture prononcé au $11^{\mathrm{e}}$ symposium annuel de l'Association pour l'avancement du service social des groupes, Montréal, 26 au 29 octobre 1989. Simone Paré en a fait la traduction.

La version anglaise de cet article doit être publiée dans un prochain numéro de Social Work with Groups.

\section{Références}

ABeLS, S.L. et P. ABELS (1980). "Social Group Works's Contextual Purposes", Social Work With Groups, vol. 3, $\mathrm{n}^{\circ} 3$ : 25-37.

Alıssı, A.A. (1982). "The Social Group Work Method : Towards A Reaffirmation of Essentials", Social Work With Groups, vol. 5, n 3 : 3-17.

Aussı, A.A. et M. CASPER (éd.) (1985). Social Work With Groups, vol. 8, nº 2. 
Breton, M. (1985). "Reaching and Engaging People : Issues and Practice Principles", Social Work With Groups, vol. 8, n 3 : 7-21.

BRETON, M. (1989). "Liberation Theology, Group Work, and the Right of Community ", Social Work With Groups, vol. 12, no 3 : 5-18. "Invitational Paper»présenté au $9^{\text {e }}$ symposium annuel sur le service social des groupes, tenu à Boston en 1987.

COHEN, N.E. (1952). "Group Work in Its Broader Implications », dans D.F. Sullivan (éd.), Readings in Group Work. New York: Association Press.

Cooley, C.H. (1962). Social Organization : A Study of the Larger Mind. New York: Sehocken Books. Edition originale, 1909, par Charles Scribner's Sons.

Coyle, G.L. (1952). "On Becoming Professional», dans D.F. Sullivan (éd.), Readings in Group Work. New York : Associative Press. (Édition originale, 1946, dans Toward Professional Standards. New York : American Association of Group Workers).

CoYLE, G.L. (1955). "Group Work as a Method in Recreation ", dans H.B. Trecker (éd.), Group Work: Foundations and Frontiers. New York: Whiteside Inc. et William Morrow and Co. (Édition originale, avril 1947, dans The Group).

DAvIS, L.E. (éd.) (1984). "Essential Components of Group Work with Black Americans", Social Work With Groups, vol. 7, n 3 : 97-109.

DeweY, J. (1922). Human Nature and Conduct. New York : Random House.

Freire, P. (1973). Pedagogy of the Oppressed. New York: The Seabury Press.

GARVIN, C.D. (1984). "The Changing Contexts of Social Group Work Practice : Challenge and Opportunity", Social Work With Groups, vol. 7, n 1 : 3-19.

Germain, C. et A. Gitterman (1980). The Life Model of Social Work Practice. New York: Columbia University Press.

Gitterman, A. et L. Shulman (éd.) (1985). Mutual Aid Groups and the Life Cycle. Itasca, III. : F.E. Peacock Publishers.

Kolodny, R. et J. Garland (1984). Guest editorial, Social Work With Groups, numéro spécial : Group Work With Children and Youth.

KUHN, T.S. (1970). The Structure of Scientific Revolutions ( $2^{\mathrm{e}}$ édition). Chicago : University of Chicago Press.

LANG, N. (1986). "Social Work Practice in Small Social Forms : Identifying Collectivity ", Social Work With Groups, vol. 9, $\mathrm{n}^{\circ} 4:$ : 7-32.

LEE, J.A.B. (1986). "Seeing It Whole : Social Work With Groups Within an Integrative Perspective", Social Work With Groups, vol. 8, n 4 : 39-50.

LeE, J.A.B. et C.R. SWENSON (1985). "The Concept of Mutual Aid", dans A. Gitterman et L. Shulman (éd.), Mutual Aid Groups and the Life Cycle. Itasca, III. : F.E. Peacock Publishers.

LEWIS, E. (1983). "Social Group Work in Community Life : Group Characteristics and Worker Role", Social Work With Groups, vol. 6, n 2 : 3-18.

Middleman, R. (1978). "Returning Group Process to Group Work», Social Work With Groups, vol. 1, $\mathrm{n}^{\circ} 1: 15-26$.

Middleman, R. (éd.) (1983). "Guest Editorial », Social Work With Groups, vol. 6, $\mathrm{n}^{\circ} 1: 3-7$.

Middleman, R. et G. Goldberg (1988). "Toward the Quality of Social Group Work Practice " : 233-246, dans M. Leiderman, M. L. Birnbaum et B. Dazzo (éd.), Roots and New Frontiers in Social Group Work, supplément $\mathrm{n}^{\circ} 3$ à Social Work With Groups. 
PÂquet-Deehy, A., E. Hopmeyer, A. Home et L. Kislowicz (1985). " A Typology of Social Work Practice With Groups ", Social Work With Groups, vol. 8, $\mathrm{n}^{\circ} 1: 65-78$.

Parsons, R. J., S.H. Hernandez et J.D. Jorgensen (1988). "Integrated Practice : A Framework for Problem Solving ", Social Work, vol. 33, n 5 : 417-421.

PARSONS, R. J. (1988). "Empowerment for Role Alternatives for Low Income Minority Girls : A Group Work Approach ", Social Work With Groups, vol. $11, n^{\circ} 4: 27-45$.

SCHOPler, J.H. et M. J. Galinsky (1984). "Meeting Practice Needs : Conceptualizing the Open-Ended Group ", Social Work With Groups, vol. 7 : 3-21.

SChWARTZ, W. (1961). "The Social Worker in the Group " : 146-177, dans The Social Welfare Forum. New York : Columbia University Press.

SChWARTZ, W. (1986). "The Group Work Tradition and Social Work Practice", Social Work With Groups, vol. 8, no 4 : 7-27.

SCHWARTZ, W. (1971). "On the Use of Groups in Social Work Practice " : 3-24, dans W. Schwartz et S.R. Zalba (éd.), The Practice of Group Work. New York : Columbia University Press.

SHAPIRO, B.Z. (1977). "Mutual Help : A Neglected Theme", Canadian Journal of Social Work Education, vol. 3, $\mathrm{n}^{\circ} 1$ : 33-44.

Shapiro, B.Z. (1986). "The Weak-Tie Collectivity: A Network Perspective", Social Work With Groups, vol. 9, n 4 : 113-125.

SHIELDS, S.A. (1986). "Busted and Branded: Group Work With Substance Abusing Adolescents in Schools", Social Work With Groups, vol. 8, n 4 : 61-81.

Shulman, L. (1984). The Skills of Helping Individuals and Groups ( $2^{\mathrm{e}}$ édition). Itasca, III. : F.E. Peacock Publishers.

TRECKER, H.B. (1955). Group Work : Foundations and Frontiers (3 édition). New York : Whiteside Inc. et W. Morrow and Co. 